\title{
Prostate Biopsy in Selecting Candidates for Hemiablative Focal Therapy
}

\author{
Matvey Tsivian, M.D., ${ }^{1}$ Marcel Hruza, M.D., ${ }^{2}$ Vladimir Mouraviev, M.D., Ph.D., \\ Jens Rassweiler, M.D., ${ }^{2}$ and Thomas J. Polascik, M.D.
}

\begin{abstract}
Focal therapy (FT) for the management of clinically localized prostate cancer (PCa) is growing from a concept to reality because of increased interest of both patients and physicians. Selection protocols, however, are yet to be established. We discuss the role of prostate biopsy in candidate selection for FT and highlight the different strategies and technical aspects of the use of prostate biopsy in this setting. In our opinion, prostate biopsy plays a major role in the selection process and tailoring appropriate treatment strategy to the patient. FT necessitates dedicated biopsy schemes that would reliably predict the extent, nature, and location of PCa in selected patients. Currently, there is insufficient scientific evidence to propose a specific biopsy scheme that could fit every candidate, providing accurate characterization of the disease in the individual patient. Further research is necessary to establish solid selection protocols that would reliably identify appropriate candidates for FT of PCa.
\end{abstract}

\section{Introduction}

$\mathbf{F}$ OCAL THERAPY (FT) for the management of clinically localized prostate cancer (PCa) is growing from a concept to reality because of increased interest of both patients and physicians. ${ }^{1}$ FT is an attractive option for appropriately selected patients because it may result in reduced morbidity and therefore better preserve quality of life compared with whole gland treatment. Appropriate candidate selection in this setting is paramount; however, the tools of selection are still debated as is their role in this setting. ${ }^{2}$

Hemiablation is today the most established focal treatment technique that aims at destroying one half of the gland while preserving the contralateral side and its neurovascular bundle. ${ }^{3}$ Therefore, appropriate candidates for this approach are represented by patients with unilateral PCa and, possibly extending to include patients with clinically insignificant contralateral disease. ${ }^{3}$

Prostate biopsy is a well-known tool in the decision-making process for the management of PCa and appears to be the most valuable criterion for selecting patients for FT. We discuss the role of prostate biopsy in candidate selection for FT and highlight the different strategies and technical aspects to propose evidence-based recommendations for the use of prostate biopsy in this setting.

\section{Is Biopsy Necessary for Focal Therapy Candidate Selection?}

Biopsy is not the only tool that plays an important role in the patient selection process for FT. Biopsy results need to be integrated with clinical, laboratory, and imaging data to be able to formulate a treatment plan. ${ }^{4}$ But can we avoid biopsy?

Currently, PCa detection is largely based on biopsy results, because imaging tools are unable to be diagnostic and achieve appropriate sensitivity and specificity levels and confirmation of PCa on biopsy is a prerequisite to further steps in planning the treatment. Moreover, tissue diagnosis provides a list of useful prognostic variables (ie, Gleason score, percent of tumor involvement, distribution of cancerous tissue within the gland, molecular markers) that may modify the treatment strategy. In the FT setting, biopsy outcomes have been shown to be one of the strongest predictors of unilateral PCa. ${ }^{2,5}$

At this time, it does not seem that biopsy can be avoided, and, therefore, the information obtained should be maximized to provide a truly useful tool for patient selection.

\section{What Is the Appropriate Biopsy Strategy?}

There is no agreement on the appropriate strategy to be used in patient selection protocols; however, it seems reasonable to suppose that the biopsy scheme used for FT patient

\footnotetext{
${ }^{1}$ Division of Urology, Department of Surgery, Duke University Medical Center, Durham, North Carolina.

${ }^{2}$ Department of Urology, Heilbronn Clinic, Heilbronn, Germany.
} 
selection should be separate from the routine office-based cancer detection biopsy familiar to every urologist.

Standard prostate biopsies are simply aimed at detecting cancerous tissue, while the requirements for the FT setting include precise characterization and mapping of the lesion(s) within the prostate. These necessary prerequisites are unavailable with routine diagnostic biopsy. Moreover, prostate biopsy is a sampling technique and, as such, suffers from sampling errors. These errors are unacceptable in the FT setting, emphasizing the need for extensive sampling and mapping to reduce the margins of error and underdetection.

The appropriate biopsy strategy for selecting patients for FT should therefore be sufficiently thorough to detect all significant cancer within the prostate, able to provide standard pathologic variables, such as Gleason grade and percent of tumor involvement per core, and able to identify core location for treatment decision and treatment planning.

From the above consideration, it is clear that the FT setting necessitates a specific and unique biopsy strategy that cannot be confused with the conventional routine cancer detection biopsy. Determining an appropriate biopsy scheme is an active field of research these days and will likely yield several options that may be safely used for the purpose of selecting candidates for FT.

\section{How many cores?}

The number of cores taken per biopsy is a crucial element for its diagnostic accuracy, given that biopsy is a sampling technique that estimates the reality and has the potential for sampling errors. Increasing the number of cores will definitely increase the diagnostic yield; however, the optimal number of cores per biopsy setting has not yet been defined.

Mayes and associates ${ }^{6}$ reported on the accuracy of routine office-based sextant biopsy in predicting unilateral PCa in low-risk patients. The authors found sensitivity and a negative predictive value of unilaterally positive prostate biopsy of $86 \%$ and $91 \%$, respectively, whereas specificity and positive predictive values were $39 \%$ and $24 \%$. Routine sextant biopsy is clearly unable to reliably detect truly unilateral PCa as determined by final pathologic analysis of radical prostatectomy specimens.

Another report compared the diagnostic accuracy of sextant vs extended biopsy schemes in all risk categories. ${ }^{7}$ It emerged that doubling the number of cores (from 6-9 in sextant to 10-20 in extended schemes) increases the diagnostic accuracy (overall correct predictions) from $45 \%$ to $59 \%$. Similarly, biopsy specificity for detecting truly unilateral PCa improved from $37 \%$ to $54 \%$, respectively. Smaller diagnostic gains could be appreciated for sensitivity and negative predictive value. The extended biopsy scheme outperformed sextant biopsy with regard to indicating truly unilateral disease; however, the ability to exclude unilateral disease was similar for both biopsy schemes. In contrast, Tareen and colleagues ${ }^{5}$ reported that among patients with unilaterally positive prostate biopsy, the likelihood of pathologically proven unilateral PCa did not vary significantly based on the number of cores obtained by biopsy.

Should the number of cores be adjusted to prostate size? The intuitive answer is yes, because biopsy is a sampling technique and the prostate gland the pool-therefore, to accurately estimate the true variables, one needs more samples from a larger pool and fewer samples from a smaller pool. A recent study by our group, however, indicates that the relationship between prostate size ${ }^{8}$ and biopsy accuracy is not a linear one, with larger $(>40 \mathrm{cc})$ prostates more frequently harboring unilateral disease enhancing biopsy performance (unpublished data), thereby suggesting that both sextant (6-9 cores) and extended (10-20 cores) biopsy techniques perform better in larger prostates with statistically significant improvement only in the latter case. More data are necessary to formulate an accurate scheme of adjustment of the number of cores to prostate size without increasing the invasiveness while maintaining diagnostic performance.

Mapping biopsy schemes that obtain an average of 50 cores have been reported ${ }^{9}$ and have been advocated as an essential tool in patient selection for FT. Obtaining these amounts of cores is likely to provide enhanced diagnostic performance of the biopsy; however, to date, there are no studies that report on diagnostic properties of these multicore techniques with radical prostatectomy specimens as the reference and no comparison with other biopsy techniques.

In summary, the optimal number of cores to be obtained on prostate biopsy to achieve adequate accuracy in detecting unilateral prostate cancer has yet to be determined. Routine office-based diagnostic biopsies did not prove reliable in predicting unilateral $\mathrm{PCa}$; however, they have an excellent power to exclude patients with bilateral disease, making these strategies appropriate for initial screening. Further research is needed to tailor the number of cores to the single patient, balancing the trade-off of diagnostic performance in detecting unilateral disease.

\section{Where to sample?}

Should prostate tissue sampling be random or should it be systematically guided by templates, and if so, which templates should be used? Unfortunately only scarce data are available on this topic in the FT setting, whereas most of the knowledge derives from routine cancer detection biopsies.

Different sampling schemes have been proposed and reported in the literature. Onik and Barzell ${ }^{9}$ and Bott and coworkers ${ }^{10}$ proposed a random saturation sampling providing more than 50 cores per prostate using the standard $0.5 \mathrm{~cm}$ transperineal (TP) grid template. These random sampling techniques maximize the number of cores and represent the most thorough prostate sampling techniques that are currently available. On the other hand, these biopsies produce a large number of tissue samples that may have different a priori probabilities to harbor cancerous tissue.

An interesting and a more elegant approach has been reported by Narayan and colleagues ${ }^{11}$ who adapted a threedimensional (3D) PCa atlas for an ultrasound-guided prostate biopsy. The atlas refers to patterns of likelihood to find $\mathrm{PCa}$ foci within the prostate according to $3 \mathrm{D}$ reconstructions of radical prostatectomy specimens. ${ }^{12}$ A further step in the same direction was reported by $\mathrm{Ou}$ and associates, ${ }^{13}$ who described the ability of the atlas-based biopsy schemes to estimate tumor volume and Gleason scores based on the location of positive biopsy by means of statistical models. This approach may reduce the random component of prostate biopsy and thus diminish the number of cores needed to reliably identify unilateral PCa and enhance the information obtained from biopsy results. Clinical application of the statistical 
model described needs to be validated in conjunction with other parameters in the setting of selecting appropriate candidates for FT, tailoring biopsy settings to the specific patient.

\section{Transrectal (TR) or TP?}

TR office-based biopsy is the standard approach, with decades of experience familiar to every urologist. TR biopsies are well-tolerated and are easily performed with the patient under local anesthesia. ${ }^{14,15}$ The current drawback is the number of cores that could be potentially obtained by a TR approach without compromising patient compliance. ${ }^{14}$

The TP approach offers the possibility to obtain multicore biopsies but requires anesthesia or sedation typically performed in an ambulatory operating room and necessitates advanced equipment that may be reflected in procedure costs. Furthermore, it has been suggested that the TP approach may be more efficient in sampling the apical and anterior portions of the prostate ${ }^{10,16-18}$ Moreover, there is less experience with the TP approach compared with the TR one; thus, urologists may be less familiar with this approach.

The morbidity profile of the TP approach differs from that of standard TR biopsies and may have a higher incidence of urinary retention episodes ${ }^{19}$; this factor has to be taken into account as well. In a direct comparison between the two approaches, Kawakami and associates ${ }^{20}$ found no significance differences in the ability to detect and characterize PCa in contrast with Emiliozzi and coworkers, ${ }^{21}$ who reported on superiority of the TP approach. The conflicting conclusions reached by these investigators may be, at least in part, because of the different number of cores taken; while Emiliozzi and colleagues $^{21}$ reported on six cores by each approach, Kawakami and coworkers ${ }^{20}$ obtained 12 and 14 cores by TR and TP approaches, respectively. These findings may suggest that the superiority of TP over the TR approach in detecting PCa may vanish when the number of cores is increased.

This hypothesis is further validated by Takenaka and associates, ${ }^{22}$ who could not find significant differences in cancer detection between the two 12-core biopsy approaches. Complications rates were compared by several authors, and no significant differences were found. ${ }^{22,23}$ A summarized comparison between the TR and TP approaches is reported in Table 1.

Table 1. A Summarized Comparison of Transrectal and Trasperineal Approach for Prostate Biopsy

\begin{tabular}{|c|c|c|}
\hline & Transrectal & Transperineal \\
\hline Setting & Office-based & Ambulatory OR \\
\hline Anesthesia & Local & Sedation/general \\
\hline $\begin{array}{l}\text { Number of cores } \\
\text { obtained }\end{array}$ & Typically $\leq 24$ & Up to over 100 \\
\hline $\begin{array}{l}\text { Sampling } \\
\text { advantages }\end{array}$ & $\begin{array}{l}\text { Peripheral, } \\
\text { posterior }\end{array}$ & $\begin{array}{l}\text { Transitional, anterior, } \\
\text { apical }\end{array}$ \\
\hline $\begin{array}{l}\text { Complications } \\
\text { rate }\end{array}$ & Low & $\begin{array}{l}\approx 10 \% \text { urinary } \\
\text { retention }\end{array}$ \\
\hline Cost & Low & High \\
\hline $\begin{array}{l}\text { Insurance } \\
\text { coverage }\end{array}$ & Yes & Problematic \\
\hline
\end{tabular}

$\mathrm{OR}=$ operating room.
Currently, there are insufficient data to form a conclusion on the superiority of one approach over the other. The TR or TP approach may be favored based on the therapy technology used; if a TP treatment is chosen (eg, cryoablation), a TP biopsy technology may be integrated on the same platform to facilitate treatment planning. The future of the biopsy approach is likely to be integrated into the treatment devices.

\section{The Importance of Mapping Biopsies}

FT aims at focal destruction of known PCa foci; thus, it is mandatory to know the location of such foci to be able to target the therapy accurately for eradication of cancerous disease while preserving healthy surrounding tissues. In this view, biopsy results should be mapped to allow for a subsequent targeted therapy to be delivered into the afflicted zones. Mapping biopsies represent the cornerstone of FT. The question is, how precisely should we map? Should every core be analyzed separately or could more cores be batched together in zones for histopathologic evaluation?

The zonal approach, dividing the prostate into a variable number of zones and batch-analyzing the cores from these zones, has been successfully used by several investigators. ${ }^{910,24}$ Another possibility is creating mathematical models that predict the exact location of the needle and create a biopsy template. ${ }^{25}$ Such mapping reduces the possibility of mapping inaccuracy because of needle displacement by deflection or by deformation of tissues. ${ }^{26} \mathrm{~A}$ real-time registration of the needle position and thus the core location has been proposed with the same intent. ${ }^{27}$ The latter approaches necessitate separate pathologic evaluation of each core, gathering detailed information that, in turn, can be introduced into the mapping software and be used as a guide for targeted therapy. This approach is likely more expensive but provides additional information both for future research and for treatment decisions regarding the individual patient, enabling truly focal therapy that would treat precisely the involved portions of the gland.

A further advancement in optimizing the processing of the biopsy cores may help to maximize the information obtained from the tissue independent of the way biopsy cores are taken from the prostate. Stock and associates ${ }^{28}$ described the selfembedding technique for prostate biopsy specimens whereby each core is placed on a saline-tinctured tissue marking the proximal (apical) and distal (basal) end immediately after removing it from the biopsy needle. If the core is fragmented, the pieces are arranged in the appropriate order. Then they are covered with a second piece of saline-tinctured tissue and separately fixed in metal clamps to avoid shifting. The closed metal clips are placed together in a formalin-filled container to be sent to the pathologist. Embedding only minimally increases the effort necessary during the biopsy procedure. For the pathologist, the procedure simplifies the process with separated, preprocessed, and embedded cores.

This technique has clear advantages for mapping biopsies and precise localization of PCa within the gland. By knowing the orientation of the cores, one can easily reconstruct the correct alignment and map the cancer with extreme precision. This method maximizes the information that is obtained by biopsy and may be of particular advantage in TP grid-guided multicore biopsies where at least two (distal and proximal) cores are taken through one grid hole. In this setting, knowing 
Table 2. Summary of Currently Available Dedicated Three-Dimensional Prostate Biopsy Systems

\begin{tabular}{|c|c|c|}
\hline Device & Manufacturer & Main feature \\
\hline TargetScan $^{\mathrm{TM}}$ & $\begin{array}{l}\text { Envisioneering Medical Technologies, } \\
\text { St Louis, MO }\end{array}$ & Software-based templates for TR approach \\
\hline Urostation $^{\circledR}$ & Koelis, La Tronche, France & $\begin{array}{l}\text { Core-location registration software on a } \\
\text { free-hand probe }\end{array}$ \\
\hline Artemis & Eigen LLC, Grass Valley, CA & $3 \mathrm{D}$ reconstruction and mapping of the cores \\
\hline Best $^{\circledR}$ Sonalis ${ }^{\mathrm{TM}}$ & Best Nomos, Pittsburgh, PA & $\begin{array}{l}\text { 3D core mapping, long probe particularly } \\
\text { useful for long prostates }\end{array}$ \\
\hline
\end{tabular}

$\mathrm{TR}=$ transrectal; $3 \mathrm{D}=$ three-dimensional.

the orientation of the cores may provide valuable information about spatial localization and extent of PCa in evaluating potential candidates for FT. A summary of currently available dedicated 3D biopsy systems is provided in Table 2.

The recent technologic advances that propose imageguidance and therefore "targeted" biopsy are promising. Targeted biopsy would therefore sample zones with an increased chance of finding and characterizing PCa foci, somewhat like mathematical models discussed. Some of the technologies investigated in this setting are: Contrastenhanced ultrasonography, elastography, and MRI/ spectroscopy that have shown intriguing preliminary results. $^{29-32}$ The implementation of targeted biopsies in the FT setting should be investigated and their potential scrutinized in larger, hopefully prospective, studies. Until these results are available, shifting from "blind" to "targeted" imageguided biopsy remains hypothetical.

Mapping biopsies are an essential prerequisite to FT implementation both today and especially in the future when more "focal" and targeted approaches may be implemented into clinical practice. For the advancement of FT and further acceptance into clinical reality of PCa treatments, mapping biopsies are essential, and detailed location of each core is recommended.

\section{Unilateral Versus Unilaterally Significant Prostate Cancer}

Should hemiabaltion be reserved only for patients with unilaterally positive cores or could patients with insignificant contralateral cancer benefit safely from FT? The answer to this question is beyond the scope of this article; however, several considerations pertaining to biopsy strategies deserve mention.

Because of needle displacement, the true position of the biopsy needle in cores that are taken near the midline may be inaccurate and induce the error of diagnosing bilateral PCa. Assuming no software guidance for the biopsy needle, this event could be more likely to occur with the TR approach because of the trajectory of the needle. This error may be detected and may be avoided by recording the exact location of the core ${ }^{27}$ or by using mathematical models that accurately predict core location. ${ }^{25}$ In addition, if there is a positive contralateral core near the midline, can the ablation be extended beyond the midline and treat this focus effectively?

The same tools in use for whole gland decision-making, summarized by Bastian and coworkers, ${ }^{33}$ will likely be used to determine the insignificance of contralateral PCa. Biopsy variables are included in most of these criteria and will need to be adapted or reformulated considering multicore biopsy schemes in the FT setting.

\section{Conclusions}

Defining appropriate patient selection protocols for FT is crucial to the success and acceptance of this approach. Prostate biopsy plays a major role in the selection process and tailoring appropriate treatment strategy to the patient. FT necessitates dedicated biopsy schemes that would reliably predict the extent, nature, and location of $\mathrm{PCa}$ in selected patients. Currently, there is insufficient scientific evidence to propose a specific biopsy scheme that could fit every candidate providing accurate characterization of the disease in the individual patient. It seems reasonable to suggest increasing the number of cores beyond the conventional 12-core scheme and tending toward a comprehensive systematic 3D mapping of the prostate in this setting. Although mathematic and "targeted" image-guided approaches to the development of efficient biopsy templates are intriguing, their clinical application has yet to be validated. Routine office-based prostate biopsy should not be relied on in selecting candidates but may serve as an initial "screening" tool that reliably identifies patients with bilateral disease.

For patients willing to embark on the option of FT, dedicated specific biopsy schemes should be requested in addition to the previously performed routine biopsy. Further research is necessary to establish solid selection protocols that would reliably identify appropriate candidates for FT of PCa.

\section{Disclosure Statement}

No competing financial interests exist.

\section{References}

1. Polascik TJ, Mouraviev V. Focal therapy for prostate cancer. Curr Opin Urol 2008;18:269-274.

2. Polascik TJ, Mayes JM, Schroeck FR, et al. Patient selection for hemiablative focal therapy of prostate cancer: Variables predictive of tumor unilaterality based upon radical prostatectomy. Cancer 2009;115:2104-2110.

3. Mouraviev V, Mayes JM, Sun L, et al. Prostate cancer laterality as a rationale of focal ablative therapy for the treatment of clinically localized prostate cancer. Cancer 2007; 110:906-910.

4. Tareen B, Godoy G, Sankin A, et al. Laterality alone should not drive selection of candidates for hemi-ablative focal therapy. J Urol 2009;181:1082-1090.

5. Tareen B, Godoy G, Sankin A, et al. Can contemporary transrectal prostate biopsy accurately select candidates for 
hemi-ablative focal therapy of prostate cancer? BJU Int 2009;104:195-199.

6. Mayes JM, Mouraviev V, Sun L, et al. Can the conventional sextant prostate biopsy accurately predict unilateral prostate cancer in low-risk, localized, prostate cancer? Urol Oncol 2009. Epub ahead of print.

7. Tsivian M, Kimura M, Sun L, et al. Predicting unilaterality of prostate cancer on routine diagnostic biopsy: Sextant versus extended. BJU Int 2009. Epub ahead of print.

8. Tsivian M, Moreira DM, Sun L, Mouraivev V, Kimura M, Moul JW, Polascik TJ. Biopsy accuracy in identifying unilateral prostate cancer depends on prostate weight. Urol Oncol (In press).

9. Onik G, Barzell W. Transperineal 3D mapping biopsy of the prostate: An essential tool in selecting patients for focal prostate cancer therapy. Urol Oncol 2008;26:506-510.

10. Bott SR, Henderson A, Halls JE, et al. Extensive transperineal template biopsies of prostate: Modified technique and results. Urology 2006;68:1037-1041.

11. Narayanan R, Werahera PN, Barqawi A, et al. Adaptation of a 3D prostate cancer atlas for transrectal ultrasound guided target-specific biopsy. Phys Med Biol 2008;53:N397-N406.

12. Zhan $Y$, Shen D, Zeng J, et al. Targeted prostate biopsy using statistical image analysis. IEEE Trans Med Imaging 2007;26: 779-788.

13. Ou Y, Shen D, Zeng J, et al. Sampling the spatial patterns of cancer: Optimized biopsy procedures for estimating prostate cancer volume and Gleason Score. Med Image Anal 2009;13: 609-620.

14. Paul R, Schöler S, van Randenborgh H, et al. Morbidity of prostatic biopsy for different biopsy strategies: Is there a relation to core number and sampling region? Eur Urol 2004;45:450-456.

15. Scattoni V, Zlotta A, Montironi R, et al. Extended and saturation prostatic biopsy in the diagnosis and characterisation of prostate cancer: A critical analysis of the literature. Eur Urol 2007;52:1309-1322.

16. Satoh T, Matsumoto K, Fujita T, et al. Cancer core distribution in patients diagnosed by extended transperineal prostate biopsy. Urology 2005;66:114-118.

17. Kawakami S, Kihara K, Fujii Y, et al. Transrectal ultrasoundguided transperineal 14-core systematic biopsy detects apico-anterior cancer foci of T1c prostate cancer. Int J Urol 2004;11:613-618.

18. Bouye S, Potiron E, Puech P, et al. Transition zone and anterior stromal prostate cancers: Zone of origin and intraprostatic patterns of spread at histopathology. Prostate 2009;69: 105-113.

19. Merrick GS, Taubenslag W, Andreini H, et al. The morbidity of transperineal template-guided prostate mapping biopsy. BJU Int 2008;101:1524-1529.

20. Kawakami S, Yamamoto S, Numao N, et al. Direct comparison between transrectal and transperineal extended prostate biopsy for the detection of cancer. Int J Urol 2007;14: 719-724.

21. Emiliozzi P, Corsetti A, Tassi B, et al. Best approach for prostate cancer detection: A prospective study on transperineal versus transrectal six-core prostate biopsy. Urology 2003;61:961-966.

22. Takenaka A, Hara R, Ishimura $T$, et al. A prospective randomized comparison of diagnostic efficacy between trans- perineal and transrectal 12-core prostate biopsy. Prostate Cancer Prostatic Dis 2008;11:134-138.

23. Miller J, Perumalla C, Heap G. Complications of transrectal versus transperineal prostate biopsy. ANZ J Surg 2005;75: 48-50.

24. Moran BJ, Braccioforte MH, Conterato DJ. Re-biopsy of the prostate using a stereotactic transperineal technique. J Urol 2006;176:1376-1381.

25. Megwalu II, Ferguson GG, Wei JT, et al. Evaluation of a novel precision template-guided biopsy system for detecting prostate cancer. BJU Int 2008;102:546-550.

26. Blumenfeld P, Hata N, DiMaio S, et al. Transperineal prostate biopsy under magnetic resonance image guidance: A needle placement accuracy study. J Magn Reson Imaging 2007;26:688-694.

27. Baumann M, Mozer P, Daanen V, Troccaz J. Towards 3D ultrasound image based soft tissue tracking: a transrectal ultrasound prostate image alignment system. Med Image Comput Comput Assist Interv Int Conf Med Image Comput Comput Assist Interv 2007;10:26-33.

28. Stock C, Hruza M, Cresswell J, Rassweiler JJ. Transrectal ultrasound-guided biopsy of the prostate: Development of the procedure, current clinical practice, and introduction of self-embedding as a new way of processing biopsy cores. J Endourol 2008;22:1321-1329.

29. Umbehr M, Bachmann LM, Held U, et al. Combined magnetic resonance imaging and magnetic resonance spectroscopy imaging in the diagnosis of prostate cancer: A systematic review and meta-analysis. Eur Urol 2009;55:575-590.

30. Pondman KM, Fütterer JJ, ten Haken B, et al. MR-guided biopsy of the prostate: An overview of techniques and a systematic review. Eur Urol 2008;54:517-527.

31. Aigner F, Pallwein L, Mitterberger M, et al. Contrastenhanced ultrasonography using cadence-contrast pulse sequencing technology for targeted biopsy of the prostate. BJU Int 2009;103:458-463.

32. Nelson ED, Slotoroff CB, Gomella LG, Halpern EJ. Targeted biopsy of the prostate: The impact of color Doppler imaging and elastography on prostate cancer detection and Gleason score. Urology 2007;70:1136-1140.

33. Bastian PJ, Carter BH, Bjartell A, et al. Insignificant prostate cancer and active surveillance: From definition to clinical implications. Eur Urol 2009;55:1321-1330.

Address correspondence to: Thomas J. Polascik, M.D.

Duke University Medical Center DUMC 2804, Yellow Zone Durham, NC 27710

E-mail: polas001@mc.duke.edu

$$
\begin{aligned}
& \text { Abbreviations Used } \\
\mathrm{FT} & =\text { focal therapy } \\
\mathrm{MRI} & =\text { magnetic resonance imaging } \\
\mathrm{PCa} & =\text { prostate cancer } \\
3 \mathrm{D} & =\text { three dimensional } \\
\mathrm{TP} & =\text { transperineal } \\
\mathrm{TR} & =\text { transrectal }
\end{aligned}
$$


